\title{
Modelo matemático para la predicción del esfuerzo de corte en el mecanizado a alta velocidad ${ }^{(\cdot)}$
}

\author{
R.E. Haber ${ }^{* * *}$, J.E. Jiménez ${ }^{*}$ A. Jiménez $z^{* * *}$ y J. López-Coronado ${ }^{* * * *}$
}

\begin{abstract}
Resumen Este trabajo presenta una primera aproximación a un modelo matemático que describe el proceso de mecanizado a alta velocidad. El modelo propuesto está sustentado en el esfuerzo de corte como variable de salida, representativa de los procesos físicos que tienen lugar durante el corte. El trabajo muestra el desarrollo matemático conducente a la obtención de las ecuaciones integro-diferenciales y los algoritmos, computacionalmente eficientes e implementados en MATLAB, para la predicción del esfuerzo de corte en el mecanizado a alta velocidad. MATLAB es un entorno de computación y desarrollo de aplicaciones, que permite realizar de forma eficiente cálculos con vectores y matrices. En el entorno, se incluyen facilidades para el análisis numérico, cálculo matricial, procesamiento de señales y visualización gráfica. En los algoritmos implementados se consideran dos tipos de útiles en el proceso de corte: fresas helicoidales, cilíndricas planas y semiesféricas. Los modelos desarrollados han sido validados experimentalmente y a través de simulaciones, corroborándose su validez y la importancia del esfuerzo de corte como variable representativa de los procesos de mecanizado a alta velocidad. Los modelos obtenidos constituyen el punto de partida para trabajos futuros relacionados con el análisis de vibraciones y su relación directa con la estabilidad en el proceso de corte y el acabado superficial.
\end{abstract}

Palabras clave Mecanizado a alta velocidad. Modelado matemático. Esfuerzo de corte.

\section{Cutting force model for high speed machining process}

\begin{abstract}
This paper presents cutting force-based models able to describe a high speed machining process. The model considers the cutting force as output variable, essential for the physical processes that are taking place in high speed machining. Moreover, this paper shows the mathematical development to derive the integral-differential equations, and the algorithms implemented in MATLAB to predict the cutting force in real time. MATLAB is a software tool for doing numerical computations with matrices and vectors. It can also display information graphically and includes many toolboxes for several research and applications areas. Two end mill shapes are considered (i.e., cylindrical and ball end mill) for real-time implementation of the developed algorithms. The developed models are validated in slot milling operations. The results corroborate the importance of the cutting force variable for predicting tool wear in high speed machining operations. The developed models are the starting point for future work related with vibration analysis, process stability and dimensional surface finish in high speed machining processes.
\end{abstract}

Keywords High speed machining. Cutting force. Mathematical modelling.

\section{INTRODUCCIÓN}

En la actualidad, una de las tareas básicas de los sistemas de fabricación es el mecanizado y, en espe- cial, el mecanizado a alta velocidad (MAV) ${ }^{[1]}$. A partir de la literatura accesible, es posible definir el MAV como el proceso de mecanizado a las máximas velocidades de corte, teniendo en cuenta las

(•) Trabajo recibido el día 25 de febrero de 2004 y aceptado en su forma final el día 26 de julio de 2004.

${ }^{*}$ ) Instituto de Automática Industrial (CSIC). km. 22,800 N-III, La Poveda. 28500. Madrid.Email: rhaber@iai.csic.es

(**) Escuela Técnica Superior. Universidad Autónoma de Madrid. Ctra. de Colmenar Viejo, km 15. 28049. Madrid.

(**) E.T.S. de Ingenieros Industriales. Universidad Politécnica de Madrid. c/ José Gutiérrez Abascal 2, Madrid 28006.

$\left(^{* * *}\right)$ Dpto. de Ingeniería de Sistemas y Automática. Universidad Politécnica de Cartagena Antiguo Hospital de Marina. c/ Doctor Fleming s/n, 30202 Cartagena. 
restricciones existentes en la pieza y el material a mecanizar, en las máquinas-herramienta y en los dispositivos de control y actuación disponibles (p.ej.:, sistemas CAD/CAM, controles numéricos). Desde un punto de vista práctico, esto implica mecanizar a velocidades de corte entre 5 y 10 veces superiores a las que se utilizan de manera convencional para cada material.

Este trabajo se centra en las fresadoras a alta velocidad en las cuales la pieza de trabajo permanece fija y el útil está acoplado a un cabezal giratorio por medio de una mordaza. A partir de dicho cabezal giratorio de altas prestaciones y de los accionamientos de cada eje se pueden alcanzar velocidades de corte muy superiores a las del mecanizado convencional, conduciendo hacia lo que se conoce, en la actualidad, como mecanizado de alto rendimiento (MAR).

El proceso de mecanizado convencional se puede considerar un proceso electromecánico complejo, en el que las fuerzas y los momentos de torsión constituyen señales representativas de los procesos físicos que tienen lugar durante el corte $\mathrm{e}^{[2]}$. El conocimiento preciso del comportamiento dinámico de estas señales permite evaluar cómo se está desarrollando el proceso de corte. De ahí, que sean de enorme utilidad en los sistemas de monitorización y control. A pesar de los grandes progresos producidos en el MAV no está clara la utilidad que pueda tener el basamento matemático, desarrollado previamente para el mecanizado convencional, en el modelado y caracterización del mecanizado a alta velocidad. Además, se requiere clarificar el papel que desempeñan en el MAV variables tales como el esfuerzo de corte, en la aportación de información relevante acerca del estado de la herramienta de corte, las vibraciones y el acabado superficial en tareas de mecanizado a alta velocidad.

Un modelo matemático es esencial para conocer el comportamiento dinámico de procesos metalúrgicos y mejorar su funcionamiento a partir del comportamiento temporal o de la respuesta frecuencial. Los modelos, tanto experimentales ${ }^{[3]} \mathrm{co}-$ mo analíticos ${ }^{[4}$ y $^{5]}$, se usan, cada vez más, en las fases de obtención, fabricación y transformación (ej.: conformado y corte) de materiales metálicos.

Hoy en día, cuando un nuevo producto o pieza metálica (p.ej.: molde) se está diseñando, antes de iniciar las etapas de realización física, éste debe haber superado las pruebas de la simulación del proceso en cuestión (ej.: el proceso de corte).

Los simuladores son programas de ordenador que predicen el comportamiento dinámico de los sistemas. Estos paquetes de software se basan en el modelo matemático de los elementos que constituyen los sistemas y en la evaluación del comportamiento de las variables más representativas. La utilidad de las simulaciones depende, en gran medida, de que los modelos matemáticos describan lo más fielmente posible el comportamiento real del proceso de corte.

Este trabajo tiene como objetivo principal obtener un modelo matemático a partir de la caracterización de los procesos físicos que tienen lugar durante el mecanizado a alta velocidad. El modelado se realizará siguiendo patrones clásicos del mecanizado convencional al considerar como variable de salida el esfuerzo de corte. De ahí que otro objetivo es estudiar y analizar el papel del esfuerzo de corte como variable en el MAV.

Este trabajo está organizado en cinco apartados. En la sección 1 se describen los antecedentes, se analizan algunos trabajos previos en la temática, se realiza una descripción rápida del mecanizado a alta velocidad $y$, finalmente, se esbozan cuestiones relacionadas con la implementación en MATLAB de dichos modelos. En la sección 2 se presenta la cinemática del modelo, considerando la forma geométrica de la hélice para cada tipo de útil: fresas cilíndricas planas y semiesféricas. En la sección 3 se aborda la formulación matemática del modelo propuesto y se plantean las ecuaciones integro diferenciales para calcular el esfuerzo de corte en el dominio del tiempo. En la sección 4 se muestran los resultados obtenidos en las simulaciones y en los ensayos en tiempo real. Finalmente, se plantean las conclusiones, eventuales trabajos futuros y líneas abiertas de investigación.

\section{ANTECEDENTES}

La caracterización del proceso de corte, a partir de modelos matemáticos que describan de manera aproximada el fenómeno físico, despertó el interés de muchos investigadores que a partir de la década de los 90 se centraron en el análisis de los fenómenos caóticos inherentes al proceso de corte ${ }^{[6]}$. Esos trabajos tuvieron continuidad y han permitido el desarrollo de herramientas computacionales para el modelado y simulación del proceso de mecanizado convencional, sustentadas en estrategias clásicas de modelado e identificación.

En la actualidad, el modelado de los procesos de mecanizado a alta velocidad y, en especial, el fresado a alta velocidad, constituye un área muy activa de investigación, que impone numerosos retos para 
la comunidad científica. En momentos que el MAV ya ha sido adoptado y es una realidad en muchas empresas, ciertos aspectos relacionados con la monitorización del estado de la herramienta ó útil de corte han sido resueltos solo parcialmente, debido en buena medida a la carencia de un modelo matemático del proceso con viabilidad para ser utilizado en aplicaciones de tiempo real. Ciertamente, el MAV es, hoy en día, una tecnología de corte con bases sólidas, que abre las puertas del mecanizado de materiales con una dureza superior a $50 \mathrm{HRc}$ o paredes delgadas de 0,2 mm.

Circunscribiéndose al MAV y, en concreto, al modelado del fresado a alta velocidad, y analizando la literatura a la que se tiene acceso, es posible comprobar que existen pocos trabajos en los que se relaciona el esfuerzo de corte como variable de salida, con las constantes y variables de entrada que definen la geometría de fresas o útiles de corte, el tipo de material a mecanizar y los propios parámetros de corte.

Este trabajo está dirigido a la obtención de un modelo matemático y la caracterización del proceso de corte a alta velocidad en operaciones de fresado a alta velocidad, a través del estudio del comportamiento dinámico del esfuerzo de corte. Como punto de partida esencial se han tenido en cuenta los trabajos precedentes relacionados con el mecanizado convencional ${ }^{[7-10]}$.

A partir de un enfoque sistémico, sustentado en la hipótesis de la viabilidad del esfuerzo de corte como variable representativa, el MAV se puede considerar un sistema de múltiples entradas y salida única (MISO). Dicho sistema involucra parámetros y variables que definen la herramienta, el material y la dinámica inherente al proceso de corte, tal y como se muestra en la figura 1 .

Por sencillez, y en una fase inicial, se plantea la necesidad de centrarse en modelos que consideren dos tipos de fresas helicoidales específicas que son de uso común en la industria del mecanizado, tanto en el sector de moldes y matrices como en la industria aeroespacial y automotriz: las fresas cilíndricas planas y las semiesféricas. En la figura 2 se muestran las características esenciales de una fresa cilíndrica plana.

\section{MODELO GEOMÉTRICO DE LA HERRAMIENTA}

El modelo geométrico a lo largo del filo de corte helicoidal incluye el análisis cinemático y dinámico del proceso de corte. La predicción de las fuerzas de corte requiere un sistema de coordenadas, el

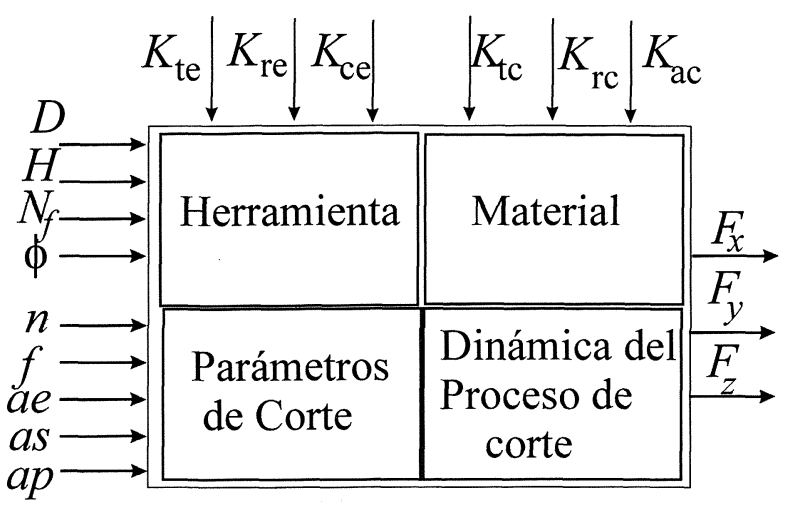

Figura 1. Diagrama del modelo del sistema visto como una caja negra.

Figure 1. Model system diagram from a black-box viewpoint.
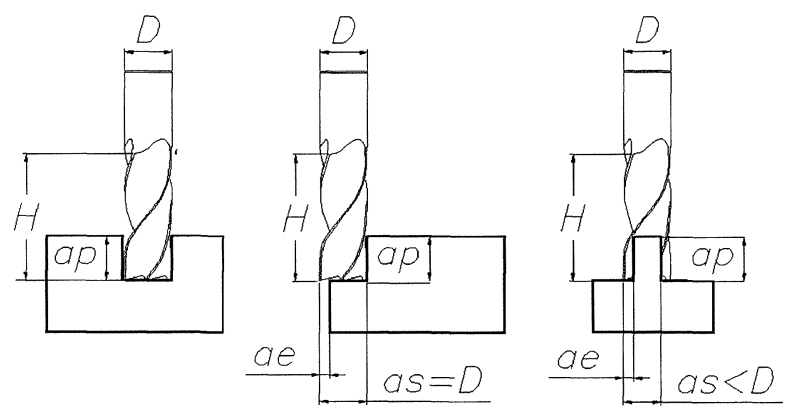

Figura 2. Fresa cilíndrica plana en régimen de corte.

Figure 2. Scheme of cylindrical end mill tool in cutting processes.

ángulo de la hélice y la distancia angular de un punto en el filo de corte ${ }^{[11]}$. Las expresiones matemáticas que definen esta geometría en un sistema de coordenadas globales se presentan a continuación en el modelo geométrico empleando una notación vectorial clásica.

El vector $\vec{r}(z)$ (Fig. 3), dibujado desde el punto $O$ hasta un punto $P$ en coordenadas cilíndricas, se expresa matemáticamente en la ecuación (1).

$\vec{r}_{j}=x_{j} \vec{i}+y_{j} \vec{j}+z_{j} \vec{k}=r\left(\phi_{j}\right)\left(\sin \phi_{j} \vec{i}+\cos \phi_{j} \vec{j}\right)+z\left(\phi_{j}\right) \vec{k}$

donde $\phi_{j}$ es el ángulo de inmersión radial de un punto $P$ en el diente $j$.

El punto $P$ se encuentra a la profundidad axial de corte $a_{p}$ en la dirección del eje $Z$, a una distancia radial en el plano $X Y$, con un ángulo de inmersión axial $\kappa(z)$ y un ángulo radial de retraso de $\psi(z)$, como se indica en la figura 3 .

Los labios helicoidales de la fresa se envuelven alrededor de la herramienta como se indica en la figura 4 . 


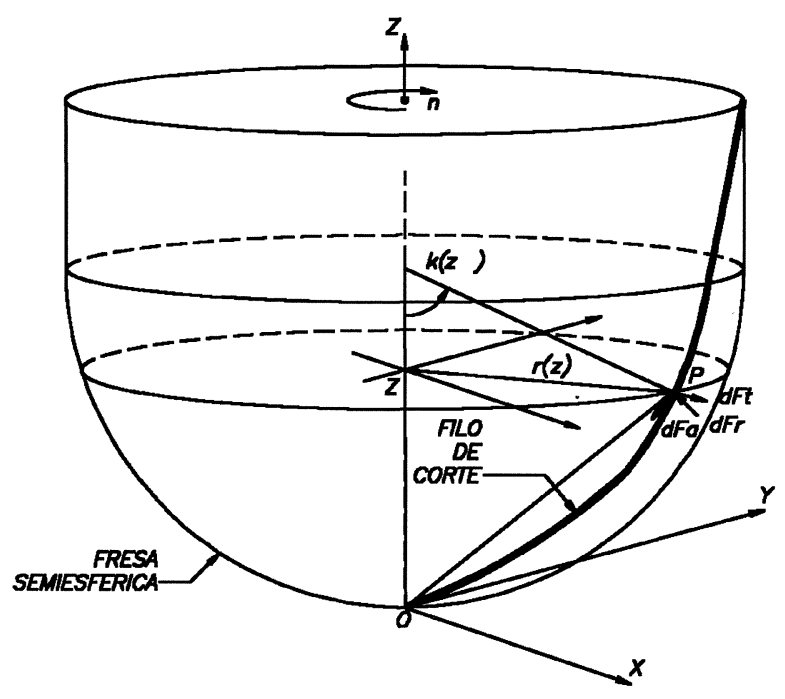

Figura 3. Geometría de la herramienta.

Figure 3. Tool geometry. Tool geometry.
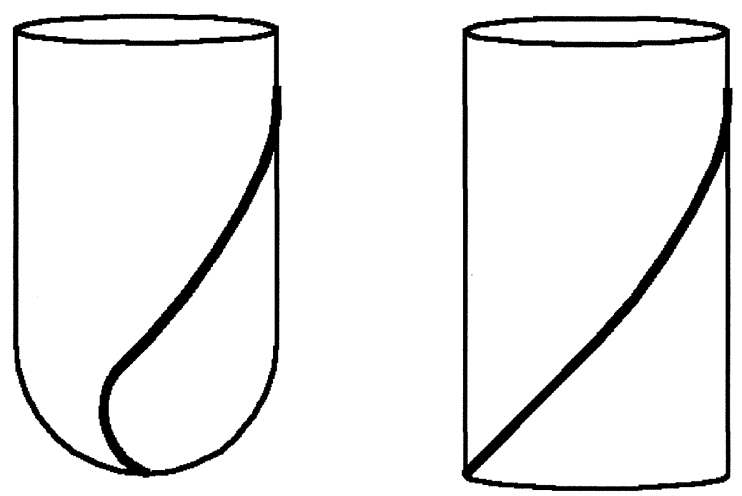

Figura 4. Filo de corte helicoidal en una herramienta de punta semiesférica y plana.

Figure 4. Helical cutting edges on the ball (left) and cylindrical end mill tools (right).

La geometría de la herramienta se representa matemáticamente considerando que el filo de corte helicoidal se envuelve alrededor de un cilindro de forma paramétrica. El modelo matemático impuesto para el borde de corte, considera que el filo se divide en pequeños incrementos en el cual los coeficientes de corte pueden variar para cada localización. Se considera como punto de referencia inicial al filo de corte de la herramienta $(j=1)$, el ángulo de rotación, cuando $z=0$ es $\phi$. El ángulo de inmersión radial para el filo de corte $j$, en cierta posición axial $z$, queda expresado como:

$$
\phi_{j}(z)=\phi+\sum_{n=1}^{j} \phi_{p}-\psi(z)
$$

El ángulo de retraso, $\psi(z)$, aparece debido al ángulo de la hélice, $\theta$. Este ángulo es constante para el caso de una fresa cilíndrica plana y varía cuando se trata de una fresa semiesférica. En el modelo generalizado para la geometría de una fresa con dientes helicoidales, el diámetro de la herramienta puede ser diferente a lo largo de esta, dependiendo de la forma de la herramienta (cilíndrica plana, semiesférica, de bola, cónica, etc.) una longitud infinitesimal de este filo de corte puede expresarse como:

$$
\begin{aligned}
& d S=|d r|=\sqrt{r^{2}(\phi)+\left(r^{\prime}(\phi)\right)^{2}+\left(z^{\prime}(\phi)\right)^{2}} d \phi \\
& r^{\prime}(\phi)=\frac{d r(\phi)}{d \phi} \\
& z^{\prime}=\frac{d z(\phi)}{d \phi}
\end{aligned}
$$

El espesor de la viruta cambia en función de la inmersión radial $(\phi)$ y axial $(\kappa)$ :

$$
h_{j}(\phi)=s_{t j} \sin \phi_{j} \cdot \sin \kappa
$$

Como se mencionó anteriormente, en este trabajo se consideran dos tipos de herramientas, fresas cilíndricas planas y con punta en forma semiesférica. Las condiciones establecidas para cada tipo de fresa se determinan matemáticamente, analizando la geometría de cada una de ellas, establecidas en los casos 1 y 2 descritos a continuación.

Caso 1: Para una fresa cilíndrica plana quedan definidas las siguientes condiciones para encontrar la solución general:

$$
\begin{aligned}
& r(z)=\frac{D}{2} \\
& \kappa=90^{\circ} \\
& \psi=k_{\theta} z \\
& k_{\theta}=(2 \tan \theta) / D
\end{aligned}
$$

Caso 2: Para una fresa con punta semiesférica el valor de se define en función del ángulo de retraso $\psi$ :

$$
\begin{aligned}
& r(z)=\sqrt{z(\psi) \cdot(D-z(\psi))} \\
& \kappa(z)=\arcsin \left(\frac{2 \cdot r(z)}{D}\right) \\
& i(z)=\arctan \left(\frac{2 \cdot \tan (\theta) \cdot \sqrt{(z \cdot(D-z))}}{D}\right)
\end{aligned}
$$




\section{MODELO DINÁMICO DE LAS FUERZAS DE CORTE}

Para determinar las fuerzas de corte en el mecanizado de alta velocidad, se considera que el corte se produce en contraposición. Los diferenciales de fuerza $\left(d F_{t}\right),\left(d F_{r}\right),\left(d F_{a}\right)$ actúan sobre un elemento infinitesimal del filo de corte de la herramienta ${ }^{[12]}$ :

$$
\begin{aligned}
& d F_{t}=K_{t e} d S+K_{t c} h_{j}(\phi, \kappa) d b \\
& d F_{r}=K_{r e} d S+K_{r c} h_{j}(\phi, \kappa) d b \\
& d F_{a}=K_{a e} d S+K_{a c} h_{j}(\phi, \kappa) d b
\end{aligned}
$$

Se considera, además, que:

$$
d b=\frac{d z}{\sin \kappa}
$$

La fresa gira a la velocidad del cabezal y la pieza de trabajo se mueve en dirección contraria a la herramienta. Con vistas a establecer las relaciones matemáticas de este hecho, se tienen en cuenta incrementos de tiempo muy pequeños. Las posiciones de los puntos a lo largo del filo de corte son evaluadas con el modelo geométrico presentado anteriormente.

Por otra parte, las características de un punto en la superficie de corte, se identifican usando las propiedades de rigidez cinemática y los desplazamientos entre la herramienta y la pieza de trabajo. Las constantes o coeficientes de corte $\left(K_{\mathrm{tc}}, K_{\mathrm{rc}}\right.$, $K_{\mathrm{ac}}, K_{\mathrm{te}}, K_{\mathrm{re} \mathrm{y}} K_{\mathrm{ae}}$ ) se pueden obtener experimentalmente a partir de los esfuerzos de corte por diente, promediados para un tipo concreto de herramienta y material ${ }^{[13}$ y 14] . Cabe señalar que estos coeficientes son muy dependientes de la localización (profundidad axial) del borde de corte. La obtención de estos coeficientes no será abordada en este trabajo.

Las fuerzas de corte se pueden evaluar empleando un sistema de coordenadas cartesianas:

$$
\left[\begin{array}{l}
d F_{x} \\
d F_{y} \\
d F_{z}
\end{array}\right]=\left[\begin{array}{ccc}
-\sin \phi \sin \kappa & -\cos \phi & -\sin \phi \cos \kappa \\
-\cos \phi \sin \kappa & \sin \kappa & -\cos \phi \cos \kappa \\
-\cos \kappa & 0 & -\sin \kappa
\end{array}\right] \cdot\left[\begin{array}{l}
d F_{r} \\
d F_{t} \\
d F_{a}
\end{array}\right]
$$

Las fuerzas totales de corte en función de se obtienen integrando la ecuación (14) a lo largo de la profundidad axial de corte para todos los labios de la fresa que están en contacto con la pieza de trabajo:

$$
\begin{gathered}
F_{x}(\phi)=\sum_{j=1}^{N f}\left(F_{x j}\left(\phi_{j}(z)\right)=\right. \\
=\sum_{j=1}^{N f} \int_{z 1}^{z 2}\left[-d F_{i j} \sin \phi_{j} \sin \kappa_{j}-d F_{i j} \cos \phi_{j}-d F_{a j} \sin \phi_{j} \cos \kappa_{j}\right] d z \\
F_{y}(\phi)=\sum_{j=1}^{N f}\left(F_{y j}\left(\phi_{j}(z)\right)=\right. \\
=\sum_{j=1}^{N f} \int_{z 1}^{z 2}\left[-d F_{i j} \cos \phi_{j} \sin \kappa_{j}-d F_{i j} \sin \phi_{j}-d F_{a j} \cos \phi_{j} \cos \kappa_{j}\right] d z \\
F_{z}(\phi)=\sum_{j=1}^{N f}\left(F_{y j}\left(\phi_{j}(z)\right)=\right. \\
=\sum_{j=1}^{N f} \int_{z 1}^{z 2}\left[-d F_{i j} \cos \kappa_{j} \quad 0-d F_{a j} \sin \kappa_{j}\right] d z
\end{gathered}
$$

donde $z_{1}$ y $z_{2}$ son los límites de integración de la zona de contacto en cada momento del corte y se pueden calcular a partir del modelo geométrico descrito anteriormente. Para el cálculo numérico, la profundidad de corte axial se divide en discos con una altura infinitesimal, $d z$. Los diferenciales de las fuerzas de corte se calculan a lo largo de la longitud del filo de corte en contacto, y se suman para encontrar las fuerzas resultantes correspondientes a cada eje $F_{x}(\phi), F_{y}(\phi)$ y $F_{z}(\phi)$ en un ángulo de rotación definido por

$$
\phi=\Omega \cdot d t
$$

donde, $\Omega$ es la velocidad del cabezal en $(\mathrm{rad} / \mathrm{s})$ y $d t$ es el diferencial de tiempo en el intervalo para la integración.

El extremo izquierdo de la herramienta es el punto inicial de referencia del ángulo de inmersión radial $(\phi)$, designado por la distancia $a_{e}$ como punto de entrada y de $a_{s}$ como punto de salida (Fig. 2). Los puntos que se encuentran entre $a_{e}$ y $a_{s}$ permanecen en los ángulos designados por: $\phi_{j}(0)=\phi+j \phi_{p} ; j=1,2 \ldots(N f)$, donde $j$ indica el labio de la herramienta.

El ángulo de inmersión para el labio, $j$, de la fresa, a causa de la profundidad de corte, a lo largo del eje $Z$ queda definido por:

$$
\phi_{j}(z)=\phi+j \phi_{p}-k_{\theta} z
$$

en la que el espesor de la viruta es:

$$
h_{j}(\phi, z)=s_{t j} \sin \phi_{j}(z) \cdot \sin \kappa(z)
$$

Las constantes de corte pueden calcularse usando la ecuación (19) a partir de la transformación del corte ortogonal en oblicuo, considerando el ángulo de la hélice como un ángulo oblicuo de corte (p.ej: $i=\theta)^{[15]}$. 


$$
\begin{aligned}
& K_{c c}=\frac{\tau_{s}}{\sin \phi_{n}} \frac{\cos \left(\beta_{n}-\alpha_{n}\right)+\tan i \tan \eta \sin \beta_{n}}{\sqrt{\cos ^{2}\left(\phi_{n}+\beta_{n}-\alpha_{n}\right)+\tan ^{2} \eta \sin ^{2} \beta_{n}}} \\
& K_{a c c}=\frac{\tau_{s}}{\sin \phi_{n} \cos i} \frac{\sin \left(\beta_{n}-\alpha_{n}\right)}{\sqrt{\cos ^{2}\left(\phi_{n}+\beta_{n}-\alpha_{n}\right)+\tan ^{2} \eta \sin ^{2} \beta_{n}}} \\
& K_{r c}=\frac{\tau_{s}}{\sin \phi_{n}} \frac{\cos \left(\beta_{n}-\alpha_{n}\right) \tan i-\tan \eta \sin \beta_{n}}{\sqrt{\cos ^{2}\left(\phi_{n}+\beta_{n}-\alpha_{n}\right)+\tan ^{2} \eta \sin ^{2} \beta_{n}}}
\end{aligned}
$$

donde, $\boldsymbol{\tau}_{\mathrm{s}}$ es el esfuerzo cortante o de cizalladura definido como el cociente entre la fuerza de cizalladura y el área plana de corte, $i$ y $\alpha_{n}$ son los ángulos oblicuo y de rebaje normal, respectivamente, $\phi_{n}$ es el ángulo normal de cizalladura o cortante, $\eta$ es el ángulo del flujo de la viruta y $\beta_{n}=\arctan (\tan$ $\beta_{\mathrm{A}} \cos \eta$ ), siendo $\beta_{\mathrm{A}}$ el ángulo de fricción.

Ciertamente, los coeficientes de corte descritos en la ecuación (19) se consideran constantes para el conjunto herramienta-material y, dichos valores, se pueden ajustar, tanto empíricamente en operaciones de fresado ó bien utilizando la transformación de corte oblicuo descrita en esta ecuación.

Las fuerzas principales se calculan en la dirección de avance $X$, normal $Y$, y axial $Z$ que son obtenidas a partir de la transformación que se indica en la ecuación (20) para el caso particular de la fresa cilíndrica plana (caso 1) ${ }^{[15]}$.

$$
\begin{aligned}
& d F_{x, j}\left(\phi_{j}(z)\right)=-d F_{t, j} \cos \phi_{j}(z)-d F_{r, j} \sin \phi_{j}(z) \\
& d F_{y, j}\left(\phi_{j}(z)\right)=+d F_{t, j} \sin \phi_{j}(z)-d F_{r, j} \cos \phi_{j}(z) \\
& d F_{z, j}\left(\phi_{j}(z)\right)=+d F_{a, j}
\end{aligned}
$$

En el caso de la fresa con punta semiesférica (caso 2), la complejidad matemática y la carga computacional se incrementa considerablemente si intentamos obtener la solución exacta. No obstante, mediante un método numérico (p. ej.: Euler, Runge-Kutta $4^{\circ}$ orden) se puede obtener una solución aproximada adecuada:

$$
\begin{aligned}
& d F_{x, j}\left(\phi_{j}(z)\right)=-d F_{i j} \sin \phi_{j} \sin \kappa_{j}-d F_{i j} \cos \phi_{j}-d F_{a j} \sin \phi_{j} \cos \kappa_{j} \\
& d F_{y, j}\left(\phi_{j}(z)\right)=-d F_{i j} \cos \phi_{j} \sin \kappa_{j}-d F_{i j} \sin \phi_{j}-d F_{a j} \cos \phi_{j} \cos \kappa_{j}(21) \\
& d F_{z, j}\left(\phi_{j}(z)\right)=-d F_{i j} \cos \kappa_{j}-d F_{a j} \sin \kappa_{j}
\end{aligned}
$$

Por otra parte, para el caso I, es posible obtener la solución exacta. Sustituyendo (17) y (18) en (15) y considerando $\kappa=90^{\circ}$ se obtiene:

$$
\begin{aligned}
d F_{x, j}\left(\phi_{j}(z)\right) & =\left\{\frac{s_{t j}}{2}\left[-K_{t c} \sin 2 \phi_{j}(z)-K_{r c}\left(1-\cos 2 \phi_{j}(z)\right)\right]+\right. \\
+ & {\left.\left[-K_{t e} \cos \phi_{j}(z)-K_{r e} \sin \phi_{j}(z)\right]\right\} d z }
\end{aligned}
$$

$$
\begin{gathered}
d F_{y, j}\left(\phi_{j}(z)\right)=\left\{\frac{s_{t j}}{2}\left[-K_{t c}\left(1-\cos 2 \phi_{j}(z)\right)-K_{r c} \sin 2 \phi_{j}(z)\right]+\right. \\
\left.+\left[K_{t e} \sin \phi_{j}(z)-K_{r e} \cos \phi_{j}(z)\right]\right\} d z \\
d F_{z, j}\left(\phi_{j}(z)\right)=\left[K_{a c} s_{t j} \sin \phi_{j}(z)+K_{a e}\right] d z
\end{gathered}
$$

La ecuación (22) se integra a lo largo de la profundidad de corte para cada labio, $j$, de la fresa:

$$
\mathrm{F}_{q}\left(\phi_{j}(z)\right)=\int_{z_{j, 1}}^{z_{j, 2}} d \mathrm{~F}_{q}\left(\phi_{j}(z)\right) d z \quad q=x, y, z
$$

Para obtener la fuerza total de corte se realiza la sumatoria de las fuerzas por cada labio de la herramienta:

$$
F_{q t o t}=\sum_{j=1}^{N f}\left(F_{q}\left(\phi_{j}(z)\right)\right.
$$

Las integraciones se llevan a cabo teniendo en cuenta que:

$$
d \phi_{j}(z)=-\frac{2 \tan \theta}{D} d z
$$

Finalmente, se obtiene la solución exacta para el caso 1 (fresa cilíndrica plana):

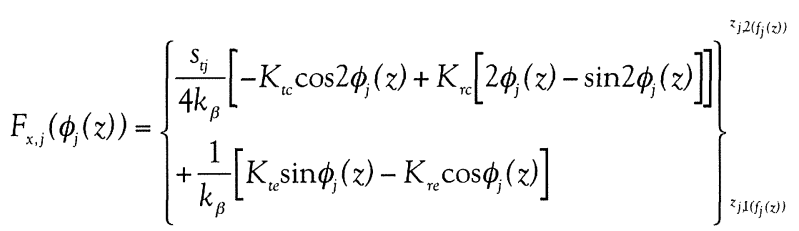

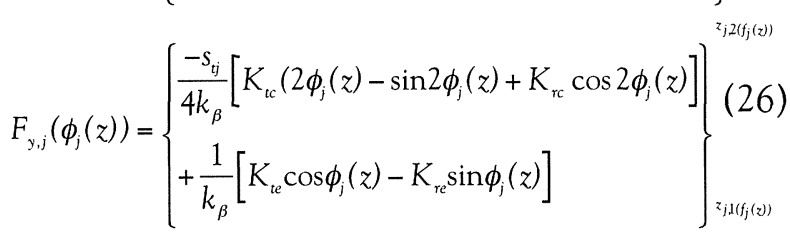

$\left.F_{z, j}\left(\phi_{j}(z)\right)=\frac{1}{k_{\beta}}\left[K_{a c} s_{i j} \cos \phi_{j}(z)-K_{a e} \phi_{j}(z)\right]_{z j 1}^{\gamma_{j i}\left(\phi_{j}(z)\right.}(z)\right)$

donde $z_{j, 1}\left(\phi_{j}(z)\right)$ y $z_{j, 2}\left(\phi_{j}(z)\right)$ son los límites inferior y superior, respectivamente, que establecen la profundidad axial de corte en el labio, $j$, de la fresa.

Los límites para la integración axial para cada labio de la fresa $\left(z_{j, 1}\right.$ y $\left.z_{j, 2}\right)$ son necesarios para calcular las fuerzas de corte. Cada segmento de arco $\left[\phi_{s t}, \phi_{e x}\right]$ de la fresa define una sección de inmersión que puede interactuar con el labio helicoidal, $j$, en cinco formas distintas. A continuación, se muestran las condiciones de borde correspondientes, que indican los puntos de intersección posibles dentro del diámetro de la fresa ${ }^{[9]}$ (Fig. 5):

Rev. Metal. Madrid 40 (2004) 247-258 


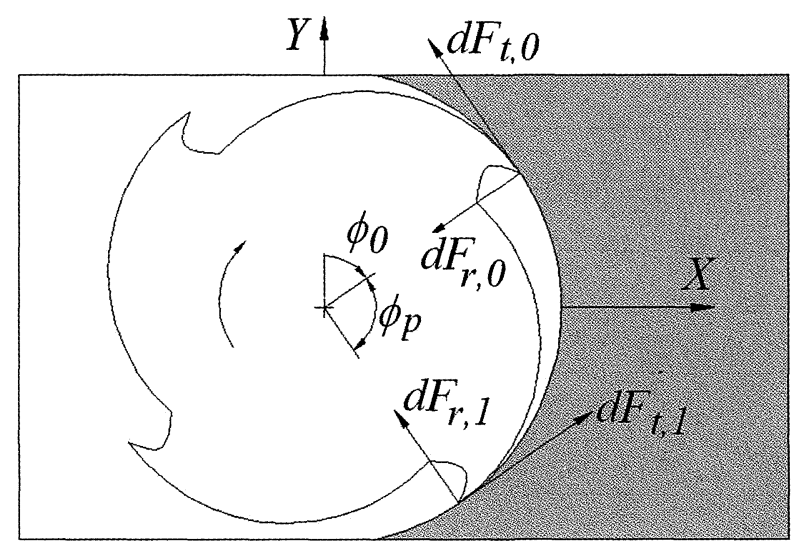

Figura 5. Componentes del esfuerzo de corte en la sección del filo de corte helicoidal.

Figure 5. Cutting force components in a section of helical cutting edge.

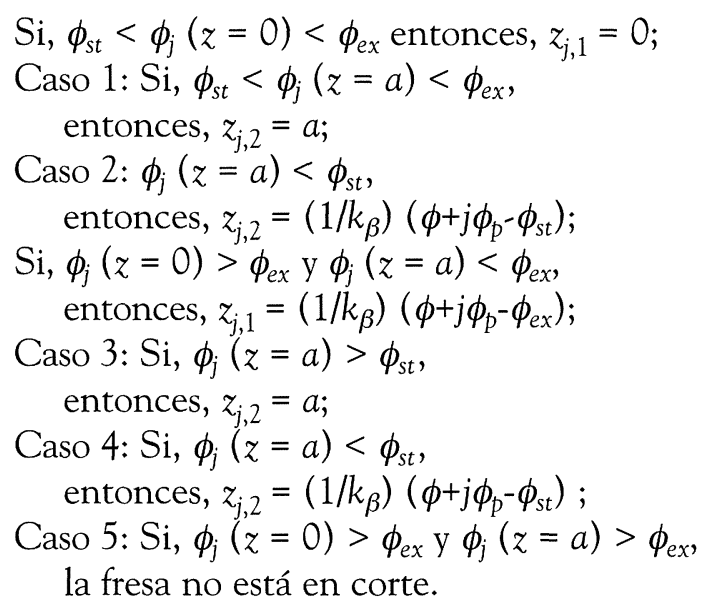

\section{SIMULACIONES Y VALIDACIÓN DE LOS MO- DELOS OBTENIDOS}

A partir de los modelos matemáticos se implementaron los algoritmos en MATLAB. MATLAB es el nombre abreviado de Matrix Laboratory. MATLAB es un entorno de computación y desarrollo de aplicaciones que permite realizar de forma eficiente cálculos con vectores y matrices ${ }^{[16]}$. En el entorno se incluyen facilidades para el análisis numérico, cálculo matricial, procesamiento de señales y visualización gráfica.

Las razones de la elección de MATLAB radican en la sencillez de la programación, la posibilidad de realizar simulaciones y aplicaciones en tiempo real, y la portabilidad de los programas desarrollados (p.ej.: existe la posibilidad de generar programas $\mathrm{C} / \mathrm{C}++$ a partir de archivos MATLAB).

A través de la simulación basada en estos modelos se pueden realizar estudios que permitan conocer la influencia de las variables y parámetros.
Las principales dificultades radican en la elección de los coeficientes de corte y las propiedades de los materiales, que han sido tomados de trabajos precedentes en el tema ${ }^{[14}$ y 15$]$. Como se mencionó anteriormente, en este trabajo no se pretende desarrollar una metodología para la obtención de dichos coeficientes sino aprovechar la información disponible en la literatura.

Los dos tipos de herramientas considerados (cilíndrica plana y semiesférica) son algunos de los más utilizados en el mecanizado de moldes y utillaje en la construcción de piezas para la industria aeroespacial y automotriz.

La propiedades del material de la pieza de trabajo, utilizadas para la simulación en el entorno MATLAB, son las correspondientes a una fundición de grafito nodular GGG-70, cuyas propiedades se indican en la tabla I.

En el estudio realizado, en la simulación y en los ensayos, reales, se consideraron dos condiciones de corte para operaciones de fresado a alta velocidad:

a) $V_{c}=546 \mathrm{~m} / \mathrm{min} ; s p=14.500 \mathrm{rpm} ; f=1.740$ $\mathrm{mm} / \mathrm{min} ; a_{\mathrm{p}}=0,5 \mathrm{~mm} ; a_{e}=0 ; a_{\mathrm{s}}=12 \mathrm{~mm}$; $\theta=30^{\circ} ; \mathrm{H}=25,0 \mathrm{~mm} ; D=12,0 \mathrm{~mm}$.

b) $V_{c}=640 \mathrm{~m} / \mathrm{min} ; \mathrm{sp}=17.500 \mathrm{rpm} ; f=2.700$ $\mathrm{mm} / \mathrm{min} ; a_{\mathrm{p}}=0,5 \mathrm{~mm} ; a_{e}=0 ; a_{\mathrm{s}}=12 \mathrm{~mm}$; $\theta=30^{\circ} ; H=25,0 \mathrm{~mm} ; D=12,0 \mathrm{~mm}$.

Las constantes utilizadas en la simulación y en la validación experimental fueron: $K_{t c}=2.172$ $\mathrm{N} / \mathrm{mm}^{2}, K_{r c}=850 \mathrm{~N} / \mathrm{mm}^{2}, K_{t e}=17,3 \mathrm{~N} / \mathrm{mm}, K_{r e}=$ $7,8 \mathrm{~N} / \mathrm{mm}, K_{a c}=726 \mathrm{~N} / \mathrm{mm}^{2}, K_{a e}=6,7 \mathrm{~N} / \mathrm{mm}$. Estas constantes o coeficientes de corte, referidos al material y a la herramienta, fueron tomados de la literatura a la que se tuvo acceso, debido a la semejanzas en las características del conjunto herramienta-material utilizado en ese estudio ${ }^{[15]}$.

\subsection{Validación experimental}

Las pruebas de validación fueron realizadas en un centro de mecanizado de alta velocidad KONDIA HS1000 equipado con un CNC abierto SIEMENS 840D. La medición del esfuerzo de corte se realizó utilizando una plataforma dinamométrica, Kistler 9257, instalada en la bancada. Las características técnicas más importantes de esta plataforma dinamométrica son: rango de medición, $[-5,5] \mathrm{kN}$ en cada eje; frecuencia natural, $>4 \mathrm{kHz}$; linealidad, $\leq 2 \%$ a escala completa; y sensibilidad, $-7,5 \mathrm{pC} / \mathrm{N}$. Se utilizaron, además, 3 amplificadores de carga 
Modelo matemático para la predicción del esfuerzo de corte en el mecanizado a alta velocidad R.E. HABER, J.E. JIMÉNEZ, A. JIMÉNEZ Y J. LÓPEZ-CORONADO

Tabla I. Propiedades mecánicas para materiales de fundición nodular de la serie GGG

Table I. Mechanical properties for materials of GGG series

\begin{tabular}{|c|c|c|c|c|c|c|}
\hline Material & & GGG40.3 & GGG40 & GGG50 & GGG60 & GGG70 \\
\hline Resistencia a la tracción & $\left(\mathrm{N} / \mathrm{mm}^{2}\right)$ & 400 & 400 & 500 & 600 & 700 \\
\hline $0,2 \%$ límite de elasticidad & $\left(\mathrm{N} / \mathrm{mm}^{2}\right)$ & 250 & 250 & 320 & 380 & 440 \\
\hline Alargamiento & $(\min . \%)$ & 18 & 15 & 8 & 4 & 3 \\
\hline Dureza Brinell & (HB30) & $120-160$ & $140-190$ & $170-220$ & $200-250$ & $235-285$ \\
\hline Módulo de elasticidad & $\left(\mathrm{N} / \mathrm{mm}^{2}\right)$ & 170 & 170 & 173 & 175 & 175 \\
\hline Resistencia a la compresión & $\left(\mathrm{N} / \mathrm{mm}^{2}\right)$ & $700-900$ & $750-950$ & $850-1.100$ & $100-1.200$ & $1.100-1.300$ \\
\hline Resistencia al cizallamiento & $\left(\mathrm{N} / \mathrm{mm}^{2}\right)$ & \multicolumn{5}{|c|}{0,9 x límite del esfuerzo de tracción } \\
\hline Densidad & $\left(\mathrm{g} / \mathrm{cm}^{3}\right)$ & 7,1 & 7,1 & 7,1 & 7,1 & 7,1 \\
\hline 0,2 \% límite de compresión & $\left(\mathrm{N} / \mathrm{mm}^{2}\right)$ & 275 & 275 & 350 & 380 & 425 \\
\hline Coeficiente Poisson & $(v)$ & 0,28 & 0,28 & 0,28 & 0,28 & 0,28 \\
\hline
\end{tabular}

5011, cada uno para medir los esfuerzos de corte $F_{x}$, $F_{y}$ y $F_{z}$. La medición se realizó por medio de una tarjeta de adquisición de datos DAQBOARD-2005 a una frecuencia de muestreo de $50 \mathrm{kHz}$.

Con el propósito de validar el modelo desarrollado, se seleccionó una fresa cilíndrica plana KAR$\mathrm{NASCH} 30.6472$, de $12 \mathrm{~mm}$ de diámetro. El material de la herramienta es micrograno $12 \%$ cobalto, recubierta 1-3 micras con TiAlN-Al, micro dureza 3.300 (HV 0,05) y máxima temperatura de $800^{\circ} \mathrm{C}$. La probeta, cuyas dimensiones son $200 \times 185 \times 50$ $\mathrm{mm}$, se escogió del material GGG-70 y se mecanizó según un patrón en espiral. Las condiciones de corte reales elegidas fueron las mismas consideradas anteriormente para llevar a cabo la simulación. Una vista general de la herramienta utilizada en los ensayos (a), la probeta y el perfil de corte (b) y el laboratorio (c) se muestra en la figura 6 .

Los ensayos en tiempo real realizados consideraron cuatro casos posibles:

a.1) $V c=546 \mathrm{~m} / \mathrm{min}: s p=14500 \mathrm{rpm} ; f=1740$ $\mathrm{mm} / \mathrm{min} ; a_{p}=0,5 \mathrm{~mm} ; a_{e}=0, a_{\mathrm{s}}=12 \mathrm{~mm}$; $\theta=30^{\circ} ; \mathrm{H}=25,0 \mathrm{~mm} ; D=12,0 \mathrm{~mm}$, usando una herramienta nueva.

a.2) $V c=546 \mathrm{~m} / \mathrm{min} ; s p=14500 \mathrm{rpm} ; f=1740$ $\mathrm{mm} / \mathrm{min} ; a_{\mathrm{p}}=0,5 \mathrm{~mm} ; a_{e}=0 ; \mathrm{a}_{\mathrm{s}}=12 \mathrm{~mm}$; $\theta=30^{\circ} ; H=25,0 \mathrm{~mm} ; D=12,0 \mathrm{~mm}$, usando una herramienta desgastada.

b.1) $V c=640 \mathrm{~m} / \mathrm{min} ; s p=17500 \mathrm{rpm} ; f=2700$ $\mathrm{mm} / \mathrm{min} ; a_{\mathrm{p}}=0,5 \mathrm{~mm} ; a_{e}=0, \mathrm{a}_{\mathrm{s}}=12 \mathrm{~mm}$; $\theta=30^{\circ} ; H=25,0 \mathrm{~mm} ; D=12,0 \mathrm{~mm}$, utilizando una herramienta nueva.

b.2) $V c=640 \mathrm{~m} / \mathrm{min} ; s p=17500 \mathrm{rpm} ; f=2700$ $\mathrm{mm} / \mathrm{min} ; a_{p}=0,5 \mathrm{~mm} ; a_{e}=0 ; a_{\mathrm{s}}=12 \mathrm{~mm} ;$ $\theta=30^{\circ} ; \mathrm{H}=25,0 \mathrm{~mm}, \mathrm{D}=12,0 \mathrm{~mm}$, utilizando una herramienta desgastada.

Los objetivos se centraron, no sólo en la validación del modelo teórico para la fresa cilíndrica plana, sino también en la comprobación de la importancia de las fuerzas de corte en el MAV y en su capacidad para aportar información relevante acerca del estado de la herramienta de corte en procesos de corte a alta velocidad. Las figuras 7, 8, 9 y 10 muestran el comportamiento real del esfuerzo de corte, $F_{x}, F_{y}$ y $F_{z}$ y la fuerza resultante, $F_{q T}$, para cada uno de los cuatro casos analizados. En líneas continuas aparece representada la respuesta del modelo para cada uno de los de los casos considerados.

Se aprecia, claramente, cómo en el caso de la herramienta nueva, el comportamiento del esfuerzo de corte en cada uno de los ejes y el esfuerzo de corte resultante estimado, $F_{e}$, se acerca bastante al comportamiento del esfuerzo de corte resultante real, $F_{q T}$. Se corrobora, además, una disminución en la magnitud del esfuerzo de corte para operaciones de corte a alta velocidad y un ligero incremento del esfuerzo de corte medio con el aumento de la velocidad de corte. En el caso de la herramienta desgastada se observa un incremento del esfuerzo de corte medio y de los valores pico en las velocidades de corte analizadas.

La tabla II muestra el estudio comparativo en cuanto al comportamiento del esfuerzo de corte resultante medio, $\bar{F}_{e}$, estimado por el modelo, y el esfuerzo de corte resultante medio, medido en una operación de corte a alta velocidad, $\bar{F}_{q T}$. La tabla muestra el comportamiento del error medio cuadrático (MSE) y del error medio absoluto (AAE). 


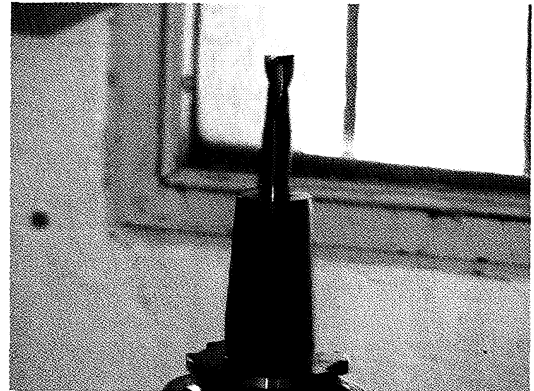

a)

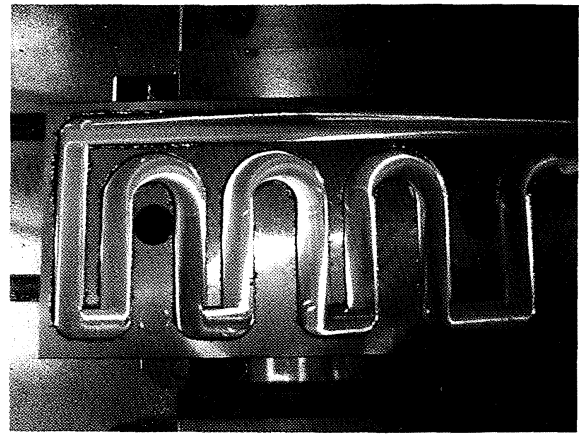

b)

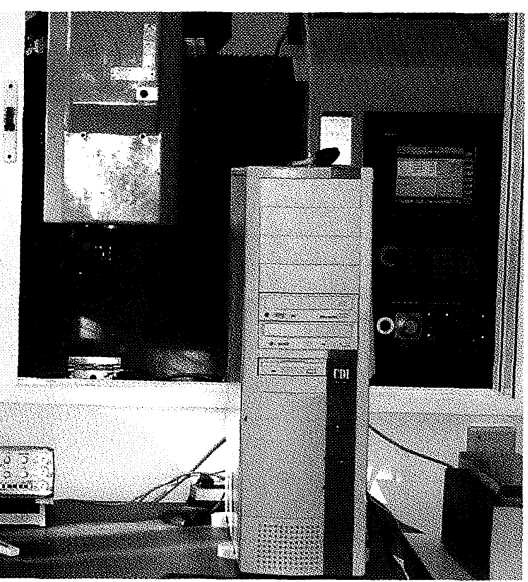

Figura 6. a) Herramienta utilizada en los ensayos; b) vista superior del perfil mecanizado en la probeta; c) vista parcial del laboratorio.

Figure 6. a) Real cutter for experiments; b) experimental piece; c) partial view of the Laboratory for machine tool research.

Tabla II. Comparación del modelo y el proceso real considerando herramientas nueva y desgastada

Table II. Comparison of cutting force model behavior and real-time measured values considering new and worn tools

\begin{tabular}{lcccccc}
\hline $\begin{array}{l}\text { Estado de la } \\
\text { herramienta }\end{array}$ & $\begin{array}{c}V_{c} \\
(\mathrm{~m} / \mathrm{min})\end{array}$ & $\begin{array}{c}F_{e} \\
(\mathrm{~N})\end{array}$ & $\begin{array}{c}F_{q T} \\
(\mathrm{~N})\end{array}$ & $\begin{array}{c}E \\
(\%)\end{array}$ & $\begin{array}{c}\text { MSE } \\
(\%)\end{array}$ & $\begin{array}{c}A A E \\
(\%)\end{array}$ \\
\hline Nueva & 546 & 56,7 & 55,6 & 4,5 & 23,4 & 6,3 \\
Desgastada & 546 & 56,7 & 82,5 & 30,2 & 50,7 & 7,6 \\
Nueva & 640 & 70,6 & 71,7 & 7,9 & 37,5 & 8,4 \\
Desgastada & 640 & 70,6 & 90,9 & 21,0 & 52,7 & 7,1 \\
\hline
\end{tabular}

Se incluye otro criterio de error, $\bar{E}=\left(\bar{F}_{q T}-\bar{F}_{e}\right) \cdot \frac{100}{\bar{F}_{q T}}$

En la actualidad, los coeficientes o constantes de corte $\left(K_{t c}, K_{r c}, K_{t e}, K_{r e}, K_{a c}\right.$ y $\left.K_{a e}\right)$ usados en el modelado se obtienen a partir del método propuesto por Budak et al. ${ }^{[14]}$, mediante una regresión lineal de las fuerzas de corte promedio para varias cargas de viruta ${ }^{[17]}$ ó mediante aproximaciones empíricas ajustadas por funciones polinómicas de
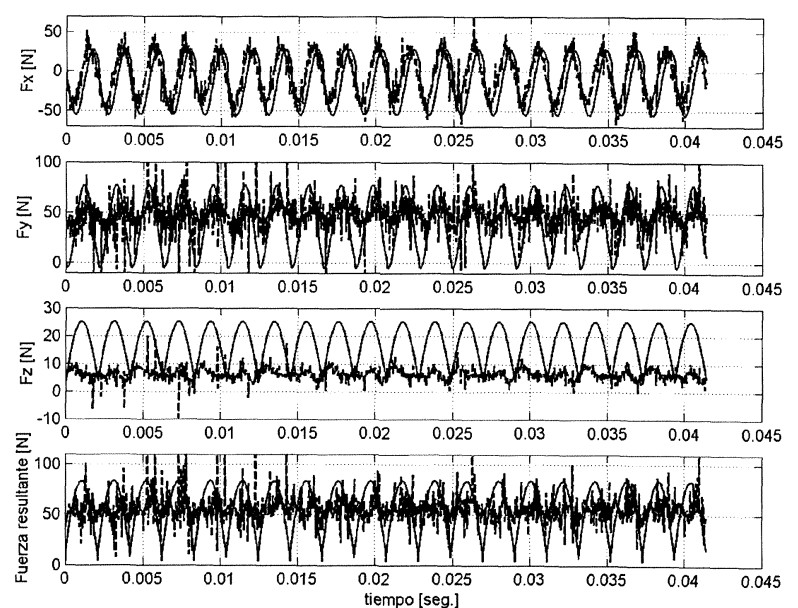

Figura 7. Simulación (línea continua) y comportamiento real (línea discontinua) de las fuerzas de corte con la herramienta nueva para el caso a.1).

Figure 7. Measured (straight line) and predicted (dashed line) cutting force for a new tool in high speed slot cutting (case a.1).

$4^{\circ}$ orden $^{[18]}$. Los coeficientes de corte utilizados en este trabajo se han tomado de la literatura ${ }^{[15]}$, 
Modelo matemático para la predicción del esfuerzo de corte en el mecanizado a alta velocidad R.E. HABER, J.E. JIMÉNEZ, A. JIMÉNEZ Y J. LÓPEZ-CORONADO
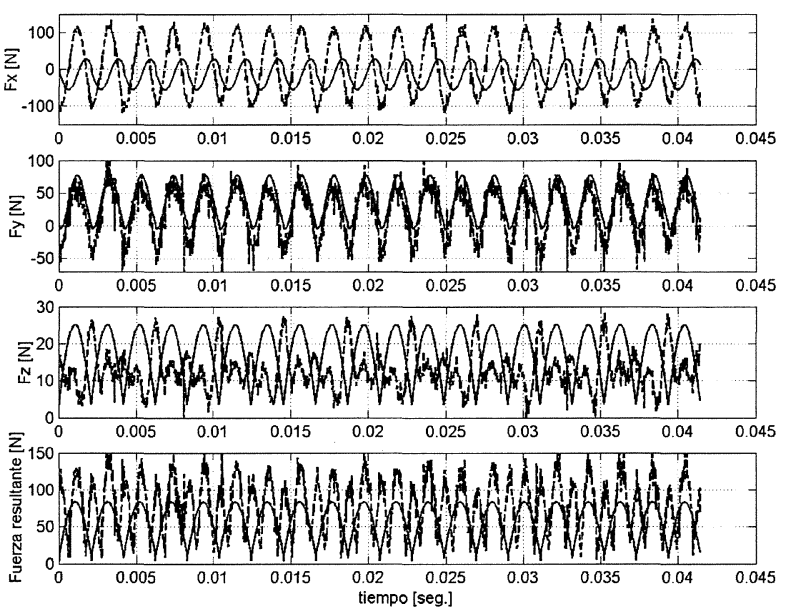

Figura 8. Simulación (línea continua) y comportamiento real (línea discontinua) de las fuerzas de corte con la herramienta nueva para el caso a.2).

Figure 8. Measured (straight line) and predicted (dashed line) cutting force for a new tool in high speed slot cutting (case a.2).
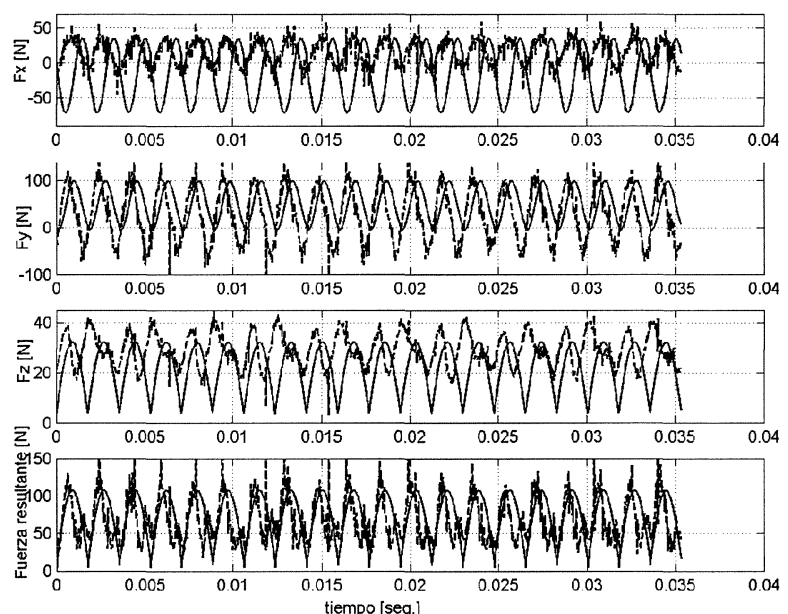

Figura 9. Simulación (línea continua) y comportamiento real (línea discontinua) de las fuerzas de corte con la herramienta desgastada para el caso b.1).

Figure 9. Measured (straight line) and predicted (dashed line) cutting force for a worn tool in high speed slot cutting (case b. 1).

por la coincidencia con el tipo de material y la herramienta utilizados en los ensayos cuyos resultados se muestran en las figuras 7-10. Ciertamente, estos coeficientes desempeñan un papel determinante en la precisión de la predicción del esfuerzo de corte. Además, la capacidad de predicción de las fuerzas de corte del modelo presentado en este trabajo también depende de coeficientes de corte obtenidos de forma experimental y, éste, continúa siendo un punto neurálgico en los modelos analíticos. Los intentos que, con mayor o menor éxito,
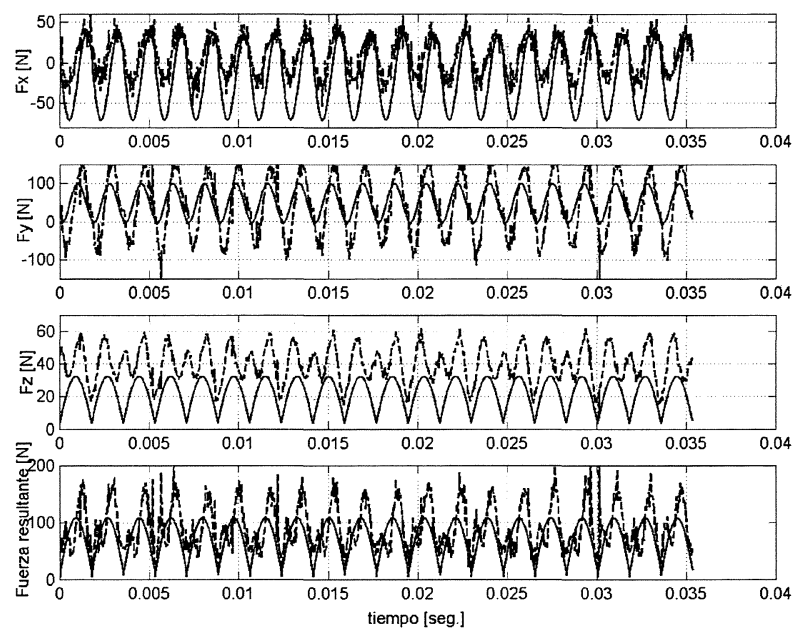

Figura 10. Simulación (línea continua) y comportamiento real (línea discontinua) de las fuerzas de corte con la herramienta desgastada para el caso b.2).

Figure 10. Measured (straight line) and predicted (dashed line) cutting force for a worn tool in high speed slot cutting (case b.2).

aparecen reflejados en la literatura, inspirados en el mecanizado predictivo, siguen dependiendo de coeficientes o constantes de corte obtenidos experimentalmente ${ }^{[19]}$.

No obstante, y analizando la literatura a la que se ha tenido acceso, este trabajo constituye el primer intento exitoso relacionado con la implementación y aplicación de algoritmos, computacionalmente eficientes, para la predicción del esfuerzo de corte en procesos de mecanizado a alta velocidad. La disponibilidad de estos modelos permite hacer frente, a corto plazo, a cuestiones esenciales relacionadas con el acabado superficial y la estabilidad de las operaciones de corte a alta velocidad.

\section{CONCLUSIONES}

En este trabajo se han obtenido los primeros resultados relacionados con el modelado del proceso de corte a alta velocidad y la validación de dicho modelo. El modelo matemático consta de dos partes fundamentales y dependientes entre sí. La primera, un sistema de múltiples entradas que define la cinemática del modelo, en la que constantes y variables describen la geometría de la herramienta, el tipo de material y los parámetros de corte. La segunda, abarca la dinámica, representada por ecuaciones integro diferenciales. Estas ecuaciones son resueltas por dos métodos. En el primer caso (fresa cilíndrica plana) se encuentra la solución analítica exacta, ya que es posible preestablecer los límites de integración así como las condiciones de 
frontera a lo largo de la geometría de la herramienta. En el caso del modelo para las fresas con punta semiesférica se aplica un método numérico (i.e., Euler), con el que se puede realizar un barrido a lo largo de la longitud efectiva de corte de la herramienta, obteniéndose una solución aproximada. Ambas soluciones difieren en la complejidad y la duración de los tiempos de cómputo.

La flexibilidad del modelo desarrollado hace que los estudios de simulación sean válidos, tanto para mecanizados convencionales como a alta velocidad, manteniendo relaciones coherentes entre los parámetros de corte como son, el avance, la velocidad del husillo y la velocidad de corte.

Por otra parte, se ha podido comprobar la importancia del esfuerzo de corte como variable representativa de los procesos de mecanizado a alta velocidad. Así, comparando los valores medios del esfuerzo de corte, se puede evaluar cuáles son las condiciones de corte de una herramienta específica, para maximizar su tasa de arranque de viruta ó maximizar la vida útil de la herramienta. De esta forma, se tendrá una mejora en los tiempos de mecanizado, vida útil de la herramienta y disminución de desechos. Asimismo, es posible obtener información necesaria para estimar el desgaste de la herramienta. Habrá que analizar, en el futuro, si aporta información suficiente sobre el estado de la herramienta de corte.

Con este trabajo y la implementación realizada en MATLAB, se abren las puertas a la utilización del modelo para predecir el acabado superficial y los lóbulos de estabilidad en procesos de mecanizado a alta velocidad.

\section{NOMENCLATURA}

D Diámetro de la herramienta [mm].

$\mathrm{H} \quad$ Altura total del filo de corte de la herramienta [mm].

$\theta \quad$ Ángulo de la hélice [grados].

$N_{f} \quad$ Número de dientes.

$f \quad$ Avance de la máquina $[\mathrm{mm} / \mathrm{min}]$.

Vc Velocidad de corte $[\mathrm{m} / \mathrm{min}]$.

$a_{p} \quad$ Profundidad axial de corte en el eje Z [mm].

$a_{e} \quad$ Profundidad radial de corte en el punto de inicio $\left(\phi_{s t}\right)[\mathrm{mm}]$.

$a_{5} \quad$ Profundidad radial de corte en el punto final $\left(\phi_{e x}\right)[\mathrm{mm}]$.

$K_{\mathrm{tc}}, K_{\mathrm{rc}}, K_{\mathrm{ac}}$ Constantes de las fuerzas de corte en sentido tangencial, radial y axial referidas al material $\left[\mathrm{N} / \mathrm{mm}^{2}\right]$.
$K_{\mathrm{te}}, K_{\mathrm{re}}, K_{\mathrm{ae}}$ Constantes en sentido tangencial, radial y axial de la herramienta $[\mathrm{N} / \mathrm{mm}]$.

$F_{x}, F_{y}, F_{z} \quad$ Fuerzas a lo largo de los ejes $X, Y, Z$ [N].

$\mathrm{F}_{\text {qTOT }} \quad$ Esfuerzo de corte total [N].

$\mathrm{F}_{e} \quad$ Esfuerzo de corte estimado por el modelo [N].

$\bar{F}_{\text {qTOT, }} \bar{F}_{e} \quad$ Esfuerzo de corte medio real y estimado por el modelo.

$r(z) \quad$ Coordenada radial de un punto del filo de corte.

$\vec{r}(z) \quad$ Vector posición.

$P \quad$ Un punto en el filo de corte.

$X, Y, Z \quad$ Coordenadas globales del sistema.

$x_{j}, y_{j}, z_{j} \quad$ Coordenadas del punto $P$ cuando está en corte.

$\psi(z) \quad$ Ángulo radial de retraso.

$\kappa(z) \quad$ Ángulo de inmersión axial.

$\phi_{\mathrm{j}}(z) \quad$ Ángulo de inmersión para el diente $j$ a la profundidad de corte $z$.

$\phi \quad$ Ángulo de rotación del filo de la fresa.

$\phi_{\mathrm{p}} \quad$ Paso angular entre dientes.

$h(\psi, \phi, z) \quad$ Espesor de la viruta.

$n \quad$ Velocidad del cabezal en $[\mathrm{rpm}]_{n}=\frac{V_{*}^{*} * 1000}{D^{*} \pi}$.

$s_{t j} \quad$ Avance por diente en $\mathrm{mm} / \mathrm{rev} s_{i j}=\frac{f}{n \cdot \mathrm{Nf}^{\prime}}$.

$\phi_{s t} \quad$ Ángulo de inmersión radial en el punto de inicio.

$\phi_{e x} \quad$ Ángulo de inmersión radial en el punto final.

i(z) Ángulo de la hélice en función de la altura.

$d F_{r}, d F_{t}, d F_{a}$ Diferencial de fuerzas en los sentidos tangencial, radial y axial.

$d z \quad$ Altura diferencial de un segmento de viruta.

dS Sección infinitesimal del filo de corte de la herramienta.

$d b \quad d S$ proyectado en la dirección de la velocidad de corte.

\section{REFERENCIAS}

[1] R.E. Haber-Guerra, A. Alique, J.R. Alique, R. HaberHaber y S. Ros, Rev. Metal. Madrid 38 (2002) 124-133.

[2] R.E. Haber, G. Schmitt-Braess, R.H. Haber, A. Alique y J.R. Alique, IEE Proc. Control Theory Appl. 150 (2003) 619-627.

[3] M. Ramirez-Mendoza, Rev. Metal. Madrid 38 (2002) 150-157.

[4] C. Camurri, Rev. Metal. Madrid 39 (2003) 132-139. 
[5] E.M. Rubio, R. Domingo, C. González y A. Sanz, Rev. Metal. Madrid 40 (2004) 46-55.

[6] S.T.S BuKKapatnam, A. LaKHTAKia y S.R.T. Kumara, Phys. Rev. E 52 (1995) 2375-2387.

[7] J.W. Sutherland y R.E. Devor, J. Eng. Ind. Trans. ASME 108 (1986) 269-279.

[8] I. Lazoglu y S.Y. Liang, J. Manuf. Sci. Eng.Trans. ASME 1 (1997) 219-234.

[9] Y. AltintAs, Manufacturing Automation: metal cutting mechanics, machine tool vibrations, and CNC design, Cambridge University Press, USA, 2000.

[10] S.A. SPIEWAK, J. Eng. Ind. Trans ASME 116 (1994) 324 330.

[11] G. Yucesan y Y. Altintas, J. Eng. Ind. Trans. ASME 1 (1996) 95-103.

[12] Y. Altintas y P. Lee, J. Manuf. Sci. Eng.Trans. ASME 120 (1998) 684-692.
[13] H.J. Fu, R.E. Devor y S.G. KaPoor, J. Eng. Ind. Trans. ASME 106 (1984) 81-88.

[14] E. Budak, Y. Altintas y E.J.A. Armarego, J. Eng. Ind. Trans. ASME 118 (1996) 216-224.

[15] S. Engin y Y. Altintas, Int. J. Mach. Tools Manuf. 41 (2001) 2195-2212.

[16] The Math Works Inc, Users manual Matlab 6.5 Release 13, Natick, Massachusetts, 2003.

[17] D. Roth, F. Ismail y S. Bedi, Comput. Aided Des. 35 (2003) 1287-1303.

[18] G.M. KIm, B.H. KIM y C.N. CHU, Int. J. Mach. Tools Manuf. 43 (2003) 917-924.

[19] H.Z. LI, W.B. Zhang y X.P. LI, Int. J. Mech. Sci. 43 (2001) 1711-1730. 